\section{MS Fundamentals Self-Paced Training, Version B}

Produced by Hewlett-Packard, Palo Alto, CA

Available through SAVANT Audiovisuals, Inc.

801 East Chapman Avenue, Fullerton, CA 92634

1993, 1995, \$595

Reviewed by O. David Sparkman

Mass Spectrometry Consultant

4045 Eagle Ridge Drive

Antioch, CA 94509

This multimedia training system consists of a VHS video tape with three separate segments, two manuals (Self-Paced Training Guide and Student Handbook), and a computer-based training program that is distributed on six diskettes and requires a little more than 10 megabytes of disk storage space. This is, by far, the best way for technically oriented people to become familiar with mass spectrometry and also get a good introduction to the use, understanding, and operation of the transmission quadrupole mass spectrometer. Although other mass analyzers and ionization techniques are reviewed nicely, the emphasis clearly is on the quadrupole mass spectrometer and electron ionization (EI).

The computer-based training (CBT) program is a well-designed set of graphic lessons that are augmented with outstanding animations and a few computer-based video clips. Reinforcement techniques make this program as entertaining as it is informative. It not only teaches the student about mass spectrometry but also how to use the program to achieve the best results.

The emphasis on EI mass spectrometry and the transmission quadrupole is accented with a good overview of other areas. Chemical ionization is given token mention. During the discussion of liquid chromatography-mass spectrometry inlets in the CBT and Student Handbook, there is a brief look at ions formed with electrospray inlets and a statement as to the "non-classical EI spectra" produced by the thermospray inlet. There is a good mix of the different ways samples are introduced into the mass spectrometer.

An outstanding feature of MS Fundamentals is the presentation of advantages and disadvantages of each option offered whenever alternatives are discussed, such as different types of high vacuum pumps, mass analyzers, and inlet systems. The material is divided into seven major categories: MS Hardware Overview; Acquiring and Evaluating Spectra; Inlets; Ion Sources; Mass Filters; Detectors; Vacuum Systems. Each learning module is divided into several lessons and a selftest.
The opening screen of the program and each learning module have an icon in the lower left corner. When selected, a full-screen definition box is displayed with prerequisites, instructions, time requirements, and additional sources of information. This attention to detail is present throughout the CBT program.

The video tape has two sections used with the MS Hardware Overview and Acquiring and Evaluating Spectra learning modules. The third part of the video is a well-done presentation of the Hewlett-Packard mass spectrometry ChemStation (DOS series).

All the hardware learning modules are well done. However, the one on Ion Sources is a real tribute to the effort that has gone into the development of MS Fundamentals. This learning module only discusses the EI source. It goes into detail as to what each component in the ion source does and why it is important. It has six lessons - Introduction, Ionization Chamber, Ionization Process, Moving Ions, Factors Affecting Spectra, Ion Source Problems - and a self-test. This is an extremely clear and thorough presentation of the EI source, which would be difficult to find anywhere else.

It is important to use both the Student Handbook and video as directed in the Self-Paced Training Guide. This is especially true in the Acquiring and Evaluating Mass Spectra learning module. If you have not covered material in all three media, you will be hard pressed to complete the self-test.

The Mass Filters learning module has an introductory lesson where transmission quadrupole, quadrupole ion trap, ion cyclotron resonance, and time-of-flight mass spectrometers are very well presented. It has two other lessons (Quadrupole Description and Quadrupole Operation) that convey an excellent understanding of the transmission quadrupole and how to optimize its performance. In the Quadrupole Description lesson, the user is taken through the development of the electrical schematic of a quadrupole mass spectrometer. This is done in such a clear way that even those with a minimal knowledge of electronics easily will understand the electronics of the quadrupole mass spectrometer. Like several others, this learning module uses a unique testing procedure during the middle of the lesson. A schematic representation of the hardware is presented with several blank label fields. When one of these blank fields is selected, a list of choices appears. After selecting one, if your choice is correct, it is entered into the box with a green background. An incorrect choice is entered into the box with a red background, and the meaning of your choice and the correct choice appears. This type of interactive learning provides reinforcement for the student.

The Master Test consists of 20 questions. You must 
score 90 or greater to complete the test. If you have incorrect answers, the final screen will not tell you what questions you missed but will indicate what areas require additional study.

The Self-Paced Training Guide shows a requirement of nine hours to complete the seven learning modules. This does not account for the time to load the software and do the Introduction section and the Master Test. The greatest benefit will be obtained when the course of study is spread over several days.

MS Fundamentals is an outstanding learning and teaching tool for use in a formal course setting or as a stand-alone self-education aid, and should become a classic in mass spectrometry training. 